\title{
El asociacionismo gallego en Buenos Aires y las posibilidades que ofrece para el estudio de la integración de los migrantes: un análisis a partir del archivo de la FAGA-MEGA *
}

\author{
Galician mutual-aid associations in Buenos Aires and its \\ significance for the study of the migrant integration: an analysis of \\ the FAGA-MEGA archive
}

Ruy Farías**

\section{RESUMEN}

Buena parte de la integración de los inmigrantes gallegos en la Argentina tuvo lugar a través de su participación en una colectividad o comunidad emigrante. La actual Federación de Asociaciones Gallegas de la República Argentina (nacida en 1921 como Federación de Sociedades Gallegas, Agrarias y Culturales), ha generado con el tiempo una valiosa masa de documentación, a la que se suman importantes fondos provenientes de múltiples sociedades gallegas de ámbito microterritorial y del extinto Centro Republicano Español de Buenos Aires. El presente trabajo busca, en primer lugar, describir someramente el asociacionismo gallego en Buenos Aires, para luego analizar la composición y características de los fondos del archivo de la Federación, ofreciendo, al mismo tiempo, una interpretación de su valor y de las posibilidades que ofrecen a la investigación.

PALAVRAS-CHAVE: Argentina. Inmigración gallega. Exilio gallego. Asociacionismo.

\section{ABSTRACT}

The integration of the Galician immigrants in Argentina was partly a result of their participation in emigrant groups or communities. Consequently, the Galician Federation of Associations in Argentina (formed in 1921 as the Galician Federation of Agricultural and Cultural Societies) has now accumulated a valuable collection of documents. It has received additional important collections from a number of Galician microterritorial associations, as well as from the now-extinct Spanish Republican Centre of Buenos Aires. In this paper, we first briefly describe the dynamics of Galician mutual-aid associations in Buenos Aires to, then, analyze the composition and characteristics of the Federations' collection and present an interpretation of its significance value and the possibilities it opens up for future investigation.

KEY-WORDS: Argentina. Galician immigration. Galician exile. Mutual-aid associations. Integration. Integración

Como sintetizara recientemente Xosé Manoel Núñez Seixas, la colectividad galaica de Buenos Aires es uno de los principales lugares de memoria de lo que fue y de lo que todavía es la emigración gallega en el mundo, pudiendo encontrarse en los

* Una versión preliminar de este trabajo fue presentada en el 5o Encuentro de Bibliotecas de Colectividades, Buenos Aires, 26 de junio de 2010.

** Doctor en Historia. Investigador del Programa de Historia Oral de la Universidad de Buenos Aires, docente de la Universidad Autónoma de Entre Ríos, y responsable del Área de Investigación del Museo de la Emigración Gallega en la Argentina. Dirección electrónica: ruygonzalofarias@yahoo.com.ar 
anaqueles de las bibliotecas de decenas de locales de sus instituciones, en sus sótanos y desvanes, auténticos tesoros de esa memoria (NÚÑEZ SEIXAS, 2009). Las páginas que siguen justificarán ambas afirmaciones, a través del análisis del valor y las posibilidades que ofrecen para la investigación los fondos documentales de la Federación de Asociaciones Gallegas de la República Argentina [en adelante, FAGA], actualmente en proceso de salvaguarda y clasificación por parte del Museo de la Emigración Gallega en la Argentina [en adelante, MEGA]. ${ }^{3}$

\section{Una Mirada a la Historiografía de la Inmigración Gallega en la Argentina y al Asociacionismo Gallego en Buenos Aires}

Argentina es el más importante destino americano de la emigración gallega. De acuerdo con las estadísticas migratorias del país austral, en torno al $17 \%$ de todos los europeos que llegaron a él entre 1857 y 1930 habría nacido en Galicia. Algo más de dos millones de españoles ingresaron en ese período al territorio argentino, de los que la mitad se habría radicado allí de manera definitiva. Teniendo en cuenta que en torno a un 55 \% de todos esos inmigrantes hispanos eran gallegos, y que tras la interrupción de los flujos migratorios a causa de la crisis económica de 1929, la Guerra Civil española de 1936 y la Segunda Guerra Mundial, una última “oleada” depositaría entre 1946 y 1960 alrededor de 110.000 gallegos más en las costas argentinas, queda un saldo de no menos de 600.000 migrantes de ese origen establecidos en el país con anterioridad al cierre del ciclo inmigratorio. Sin embargo, su acusada tendencia a concentrarse en las zonas urbanas del Litoral pampeano, y muy particularmente en Buenos Aires, generó un fenómeno aún más impactante: que a lo largo de buena parte del siglo pasado viviesen en la ciudad porteña más gallegos que en ningún otro núcleo urbano del planeta. ${ }^{4}$

Gracias a la atención que desde mediados de la década de 1980 le han dedicado historiadores y otros científicos sociales, contamos hoy con una masa crítica de estudios de caso (y algunas obras de síntesis) que han permitido la comprensión de los factores macroestructurales y microsociales del fenómeno migratorio gallego a la Argentina, el conocimiento de la inserción espacial y laboral del grupo en su principal destino rioplatense (Buenos Aires y su periferia), su obra socioeducativa en la urbe porteña, la

\footnotetext{
3 En diciembre de 2003 la FAGA dispuso la creación del MEGA, que fue inaugurado oficialmente el 9 de diciembre de 2005, y desde entonces se encuentra abierto a la comunidad gallega y al público en general.

4 De hecho, ya en 1914 moraban en Buenos Aires unos 150.000. Por la misma época, la ciudad más importante de Galicia (A Coruña) apenas contaba con 60.000 habitantes. Vid. Moya (2004), Villares y Fernández (1996), Fernández y Moya (1999). Para una mirada de conjunto sobre la inmigración europea en la Argentina, Devoto (2003).
} 
dinámica política y cultural de su asociacionismo, la aparición y desarrollo de identidades alternativas u opuestas a la española, las características de sus dirigencias y liderazgos, las imágenes, estereotipos, prejuicios y formas latentes o concretas de xenofobia, el rol dela mujer, etc. ${ }^{5}$

Sin embargo, la mayor parte de los trabajos se han concentrado en el caso de Buenos Aires y en la etapa de inmigración "masiva" (aprox. 1880-1930), mientras que los pocos que superaron la línea 1930/ 1936 por lo general se orientaron bien al estudio del exilio republicano, bien al de los condicionantes y efectos de las políticas migratorias del régimen franquista, en relación con las ideas, metas e iniciativas del gobierno argentino (DE CRISTÓFORIS; FERNÁNDEZ, 2008, p. 77-78). ${ }^{6}$ Así, poco o nada informan sobre las características generales de la "última oleada" entre 1946 y 1960, período en el que la Argentina fue nuevamente el principal destino americano de la emigración gallega (y española) a Latinoamérica. De ese modo, nuestros conocimientos de esa inmigración resultan escasos, fuera de la certeza de que la misma se hallaba motorizada por una motivación económica inmediata (producto de una economía incapaz de absorber la mano de obra excedentaria que generaban las áreas rurales), y de que entrañó un amplio proceso de reagrupación familiar, según se deduce de la mayor emigración femenina y la menor tasa de retorno en comparación con los estándares del período 1880-1930 (FERNÁNDEZ VARGAS, 1992; PALAZÓN FERRANDO, 2005). ${ }^{7}$

Después de encontrar donde vivir y un modo de ganarse la vida, el paso siguiente en la adaptación del inmigrante consiste en recrear una red social secundaria. Con ese fin los gallegos fundaron o se afiliaron a una plétora de asociaciones voluntarias de corte étnico, tanto a las genéricamente españolas como a las específicamente gallegas. De este modo, buena parte de la integración de los inmigrantes galaicos en la Argentina tuvo lugar a través de su participación en una colectividad o comunidad emigrante, que conforma un espacio de interacción social en el que se recrea aquél del que proceden sus integrantes.

\footnotetext{
${ }^{5}$ Sin pretensiones de exhaustividad, resultan de ineludible consulta las obras de Sánchez-Albornoz (1988), Clementi (1991), ;Vives, Vega y Oyamburu (1992), Marquiegui (1993), Núñez Seixas (1992, 1998, 2001, 2002), Palazón Ferrando (2005), Sánchez Alonso (1992, 1995), Cagiao Vila (1997, 1999), Duarte (1998), Vázquez González (2009), Da Orden (2005), Núñez Seixas y Soutelo Vázquez (2005), Bernasconi y Frid (2006), Cagiao Vila y Núñez Seixas (2007), Farías (2007), Lojo (2008), De Cristóforis y Fernández (2008), así como también el trabajo en prensa de García Sebastiani. Por otra parte, es necesario añadir a esta lista los legados extraordinarios de Vilanova Rodríguez (1966), Pérez-Prado (1973), Palmás (1978) y Cupeiro (1989).

${ }^{6}$ Véanse, por ejemplo, los de Senkman (1991), Quijada Mauriño (1989), Schwarzstein (2001), Devoto (2001), Fernández Vicente (2005) y Galante ( 2005).

${ }^{7}$ Con todo, pueden citarse algunos capítulos incluidos en varias de las obras antes citadas, y también alguna otra pronta a parecer, como la de De Cristóforis.
} 
La fundación de la primera sociedad de inmigrantes españoles genérica en el país (la Sala Española de Comercio) data de 1852. Si bien la misma desapareció un lustro más tarde, en 1857 surgieron el Club Español, la Asociación Española de Socorros Mutuos de Buenos Aires [en adelante, AESMdeBA] y la Sociedad Española de Beneficencia. Hacia 1914, éstas y otras asociaciones hispánicas de ayuda mutua se habían convertido en un enorme aparato institucional, existiendo por entonces en el país un total de 250 con 110.000 socios, lo que implica que 13 de cada 100 españoles se hallaban mutualizados. Ahora bien, teniendo en cuenta que los gallegos representan a lo largo del período 1860-1960 la mitad de todos los inmigrantes españoles llegados a la Argentina, parece lícito afirmar que detrás de la denominación de "española" de una sociedad cualquiera, solía ocultarse una mayoría gallega entre sus dirigentes y afiliados.

Pero, por otra parte, dentro de este contexto pan-hispánico afloró también un rosario de instituciones propiamente galaicas. Los gallegos desarrollaron en la Argentina prácticamente todas las posibilidades de asociacionismo étnico, combinando la procedencia geográfica (regional, provincial, local, comarcal o parroquial) con los objetivos específicos que cada institución perseguía (mutualistas médicas, instituciones de beneficencia, centros culturales, recreativos, deportivos, etc.). Al proceder la mayoría de ellos del medio rural, sufrían al desembarcar en la populosa y cosmopolita Buenos Aires de comienzos del siglo XX (muchas veces sin siquiera haber visitado antes la capital de su provincia) un importante choque cultural. Ese contraste los empujaba a la solidaridad étnica con sus coterráneos, a fin de amortiguar el impacto y acomodarse mejor a las nuevas condiciones mediante la ayuda mutua prestada por las sociedades étnicas. Pero del mismo modo, la experiencia de la emigración contribuyó a cambiar la mentalidad de muchos de los que cruzaban el océano, en un proceso de dimensiones tanto individual como colectiva. Así, el campesino que emprendía el camino de la emigración descubría un mundo urbano y de servicios, donde la movilidad social ascendente se convertía en una opción real y accesible. Y también uno nuevo de relaciones sociales, encuadrado en experiencias diferentes de confrontación de clase y de oficio. Esta toma de conciencia influyó igualmente en que valorase las ventajas de la asociación y, por tanto, fundase sociedades de ámbito gallego y de ámbito comarcal y local (CAGIAO VILA, 1999; NÚÑEZ SEIXAS, 1999a, 1999b, 2000).

Aunque el asociacionismo mutualista gallego en la Argentina tiene precedentes genéricos en el período colonial, su eclosión coincidió con el comienzo de la emigración transoceánica masiva, en las últimas dos décadas del siglo XIX. En 1879 se fundó en Buenos Aires el primer centro gallego de la Argentina, pero el mismo tuvo una vida breve vida, ya que se extinguió en 1892. Tras la desaparición de aquel primer Centro 
Gallego de Buenos Aires, la capital argentina no contó con ninguna gran sociedad mutualista de ámbito galaico por 15 años. ${ }^{8}$ Sin embargo, coincidiendo con el arribo de las grandes oleadas de inmigrantes galaicos entre 1904 y el estallido de la guerra europea, nacería un nuevo y definitivo Centro Gallego de Buenos Aires (1907), que en la década de 1930 superó en número de socios a la AESMdeBA, ${ }^{9}$ y se convirtió en la entidad mutual más grande de la Argentina y de la América hispana. Y junto a él, brotaron a lo largo de las décadas siguientes varias sociedades regionales (asilos, centros culturales, políticos y otros por el estilo), cuatro centros provinciales (uno para cada provincia) y un sinfín de asociaciones microterritoriales. ${ }^{10}$

Con ellas la expansión de las sociedades españolas y gallegas adquirió una nueva dimensión. A partir de la fundación en 1904 de la primera sociedad de instrucción conocida en Buenos Aires, ${ }^{11}$ surgió todo un enjambre de pequeñas instituciones gallegas que constituyen, indudablemente, uno de los fenómenos que particularizan a la colectividad galaica dentro del contexto del asociacionismo hispánico. ${ }^{12}$ Estas formas asociativas polivalentes, adoptaban como marco espacial de actuación entidades geográfica-administrativas propias del país de origen, como la parroquia, el municipio o la comarca. A veces también el partido judicial o distrito. Es decir, ámbitos territoriales de relación e interacción social inferiores a la provincia, que constituían verdaderas esferas de recreación del espacio social. Las solidaridades locales pervivieron en la otra orilla del océano, y fueron en un comienzo de carácter más inmediato y vinculante que las "regionales" (gallega) o "nacionales" (española), lo que reflejaba -y al mismo tiempo tenía su causa- en la propia estructura de las cadenas migratorias y en la reproducción de las redes de paisanaje en la Argentina para procurar colocación laboral, protección inmediata al recién llegado, espacios para el esparcimiento, etc.

En términos generales, el papel jugado por las instituciones de la colectividad como ámbitos de socialización de los inmigrantes es un hecho probado. Al intentar reproducir pautas y mantener costumbres de la propia sociedad de origen, constituyeron los mecanismos más evidentes de expresión de la identidad. Ciertas

8 En cambio, surgieron algunas asociaciones recreativo-culturales (como los diversos orfeones), la Unión Gallega (fundada en 1900), etc.

939.118 en 1932, 57.000 en 1938, llegando en el período 1961-1962 a su máximo histórico: 104.855.

${ }^{10}$ Esta terminología pertenece a Peña Saavedra (1991, v. 1, p. 355-388).

${ }^{11}$ Como puntualiza Núñez Seixas (2001, p. 356, 375-378), en puridad, la denominación de sociedades de instrucción se reserva sólo a aquellas asociaciones microterritoriales que tenían como objeto la creación o dotación de establecimientos escolares en sus lugares de origen. Su objetivo implícito era contribuir a la regeneración individual del campesino, hacerlo conciente de sus derechos y prepararlo para luchar por su emancipación, capacitarlo para la acción colectiva y así dar fin al caciquismo y fanatismo religioso que invalidaría hasta entonces sus derechos democráticos.

12 Tan sólo en la capital argentina y entre 1904 y 1936, habrían existido no menos de 327 sociedades de instrucción, indicando las últimas estimaciones elaboradas a partir de la gran base de datos del Arquivo da Emigración Galega para todo el país la presencia de 476 entre 1901y 1933. 
noticias difundidas por los órganos de prensa de las mismas, anunciando enlaces matrimoniales, nacimientos, efemérides, defunciones, viajes y cambios de residencia, son un expresivo reflejo de eso. En el caso particular de las mujeres, con una dedicación laboral que se desarrolló mayoritariamente en el ámbito doméstico, alcanzaron todavía mayor importancia este tipo de relaciones. ${ }^{13}$ La pervivencia de esas solidaridades locales y el alto promedio de retornos (al menos hasta el estallido de la Guerra Civil española), junto al generalmente corto período de estancia de los emigrantes allende el mar (siempre previamente a la coyuntura bélica de 1936-1939), fueron factores que favorecieron el mantenimiento de los vínculos con las comunidades parroquiales o municipales de origen. Y estos lazos se canalizaron y cristalizaron, principalmente, a través de la orientación de las sociedades de emigrados en la Argentina, que a partir de comienzos del siglo XX incluirán entre sus objetivos no sólo el socorro mutuo para sus miembros, sino también la coordinación de esfuerzos para llevar a cabo iniciativas diversas en el país de origen.

Desde el punto de vista de su evolución y naturaleza sociopolíticas, las sociedades microterritoriales presentan características muy diversas, pero la mayoría de ellas exhibían un ideario político-social bastante moderado, vinculado ante todo con un proyecto democrático-progresista de imprecisos contornos y centrado en un principio en problemáticas relacionadas con el poder local, que en general es definible como anticaciquil, de construcción de la sociedad civil y de regeneración. ${ }^{14} \mathrm{La}$ fundación o dotación de establecimientos educativos en sus lugares de origen fue unas de los fines más importantes de estas sociedades, y se plasmó abundantemente en varias zonas de Galicia. ${ }^{15}$ Sin embargo, ese no era su único fin. El objetivo implícito en la educación había de ser contribuir a la regeneración individual del campesino, hacerlo consciente de sus derechos y capaz de luchar por su emancipación, capacitarlo para la acción colectiva y así acabar con el caciquismo y el fanatismo religioso que invalidarían hasta entonces (según el pensamiento de los precursores de estas iniciativas) sus derechos democráticos. ${ }^{16}$ Muchas de esas pequeñas instituciones conservaron una larga

\footnotetext{
${ }^{13} \mathrm{El}$ acceso a las sociedades como lugares de contacto externo fue a veces la única posibilidad de facilitar las relaciones personales para muchas gallegas, lo que incidirá en el comportamiento endogámico, sobre todo mientras no se produjo la incorporación de la mujer a otro tipo de trabajos.

${ }_{14}$ Para una explicación pormenorizada de esos fines, vid. Núñez Seixas (1998, p. 109-159).

${ }^{15}$ Se ha calculado en 326 el total de escuelas gallegas construidas, dotadas y/ o mantenidas gracias a la financiación de las sociedades de instrucción de América.

$16 \mathrm{Al}$ mismo tiempo, como queda dicho, también jugaban un papel relevante los fines mutualistas, pero éstos irán perdiendo importancia a medida que las grandes instituciones benéficoasistenciales, como el Centro Gallego porteño, se vayan asentando y desarrollando, de modo que las sociedades microterritoriales terminarán concentrándose a la larga en las actividades recreativas.
} 
vida (llegando algunas incluso hasta nuestros días), pero a partir de 1939 sus posibilidades de actuación a favor de sus comunidades locales de origen se circunscribieron (en razón de la nueva situación política española) prácticamente al campo de la beneficencia. ${ }^{17}$ Aún así continuaron (o continúan) jugando un papel de lugar de sociabilidad para los emigrantes, integrando en muchos casos a los hijos y nietos de los socios que no retornaron a Galicia.

Por otra parte, el rol que las sociedades microterritoriales gallegas de la Argentina jugaron en la dinámica social y política de su país de origen, no impidió que surgiesen proyectos de ámbito gallego que superaban el localismo de la actuación de cada una de ellas. Estos se plasmaron en iniciativas comunes de apoyo al movimiento agrarista galaico, y también en proyectos federativos que se sucedieron a lo largo de las décadas de la segunda y tercera década del siglo XX. En 1921, como producto de la coalición de varias sociedades gallegas de ámbito microterritorial (de las que llegaría a reunir más de medio centenar a mediados de la década de 1950), surgió en la capital argentina la Federación de Sociedades Gallegas, Agrarias y Culturales [en adelante, FSG]. ${ }^{18}$ Influenciada primero por el socialismo y el agrarismo, y más tarde por el nacionalismo gallego y el comunismo, hizo gala constantemente de un alto grado de compromiso con Galicia, manifestado en su clara vocación de intervención en la modernización de su sociedad y vida política. Esa vocación se concretizó al principio en su apoyo a la lucha del campesinado gallego por librarse de algunos de sus lastres decimonónicos (como el caciquismo imperante en el medio rural, o el sistema foral). Más tarde, y pese a sufrir una escisión en 1929, fue capaz de intervenir de manera decidida en la política gallega durante la IIa República española, hasta el punto de enviar delegados propios que participaron en las elecciones apoyando candidaturas republicanas y galleguistas, algunos de los cuales llegaron incluso a alcanzar el rango de diputados en el parlamento español. Tiempo después, la FSG apoyó la campaña a favor de un Estatuto de Autonomía para Galicia y, en la coyuntura de la Guerra Civil española, desplegó una actividad frenética, llegando a crear en su seno una organización específica para apoyar su esfuerzo de guerra del legítimo gobierno español. Apoyo que no acabó con la derrota en 1939, sino que continuó a favor de los exiliados y refugiados que el conflicto generó y, en ocasiones, también de los represaliados por el franquismo. De hecho, hasta la llegada de la denominada “transición española” en la segunda mitad de la década de 1970, la FSG albergó a varios

${ }^{17}$ Con todo, en el caso de la sociedad Pro-Escuelas de Ferreira (San Sadurniño, A Coruña), estudiado por Núñez Seixas y Soutelo Vázquez (2005, p. 135-156), sus afiliados continuaron contribuyendo al sostenimiento del edificio escolar hasta entrada la década de 1960.

${ }^{18}$ A mediados de la década de 1950 la FAGA contaba con unos 15.000 afiliados. Para una historia de la misma, véase Díaz (2007). 
de los elementos más combativos del galleguismo y el republicanismo emigrante y exiliado, y encarnó como quizás ninguna otra institución hispana de la Argentina la memoria republicana, la oposición a la política franquista, y la lucha por el retorno de la democracia a España. ${ }^{19}$

Por último, desde comienzos de la década de 1930, y como consecuencia de la importante caída de los flujos migratorios a la Argentina, se advierte en la colectividad gallega la desaparición de algunas sociedades y un proceso de unificación de otras de un mismo ámbito territorial, proceso que se verá acelerado durante y después de la Guerra Civil. Así, a lo largo de la década de 1940 se constituyen el Centro Orensano (1941), el Centro Pontevedrés y el Centro Lucense (1942) y, finalmente, el Centro Coruñés (1950), que a comienzos de la década de 1980 los cuatro centros provinciales se refundirán en un único Centro Galicia de Buenos Aires.

\section{Algunas Consideraciones en Torno al Valor del Archivo de la FAGA-MEGA}

Debido a lo ya comentado, con el paso del tiempo la FSG (hoy denominada FAGA) ha generado una enorme y valiosa masa documental propia. Sin embargo, su archivo alberga también los fondos de varias docenas de sociedades federadas, y una parte sustancial de la documentación producida a partir de la primera década del siglo XX por el desaparecido Centro Republicano Español de Buenos Aires [en adelante, $\mathrm{CRE}]$. Si bien la tarea de organización de estos materiales comenzó mucho antes de la creación del MEGA (2005), ${ }^{20}$ las más de 800 unidades de conservación ya localizadas, integradas por registros de socios, libros de actas de asambleas y comisiones directivas, carpetas de documentación contable, biblioratos de correspondencia enviada y recibida, cajas de folletería, etc., que suman ya más de 80.000 documentos, se encuentran ahora en proceso de sistemática clasificación, puesta en valor y digitalización. ${ }^{21}$

$\mathrm{Si}$ resulta pertinente afirmar que muchas de las sociedades gallegas de la Argentina conservan auténticos tesoros de la memoria de lo que fue y aún es la inmigración gallega en dicho país, ello se debe, en primer lugar, a que los documentos

19 No obstante, la Federación distó de ser la única institución gallega comprometida con la causa republicana y democrática. Por el contrario, existió en la colectividad gallega de la Argentina toda una red de comités de auxilio a la República, a veces a escala municipal o comarcal, los cuales hicieron en ocasiones suscripciones para enviar fondos a algún coterráneo del que se conocía su situación en los campos de refugiados del país galo.

20 El primer intento de organizar este archivo del que tenemos constancia data de mediados de la década de 1990, pero el mismo fue muy limitado y no siempre redundó en una mejor conservación de los materiales tratados.

${ }^{21}$ El MEGA ha puesto en marcha varios proyectos de conservación del Programa de Colecciones, con la finalidad de salvaguardar el patrimonio que preserva y avanzar con los Programas de Documentación, Exposición e Investigación. Sobre los mismos, véase Chiloteguy (2010). 
que estas instituciones han producido tienen un importante valor inmaterial. Los mismos se encuentran "atados a las vivencias de una comunidad, a su posición dentro de una sociedad de inmigrantes, a su autoestima y orgullo", lo que les da un valor "no en sí, sino en función del significado otorgado por un colectivo." Testimonian

[...] aquellos tiempos en que los abuelos y bisabuelos de los que en Galicia vivimos tuvieron que ir a ganar el pan y una vida mejor a Argentina. En que los recursos se ubicaban en el Río de la Plata, igual que el progreso y la modernidad, y las necesidades se situaban en Europa. En que los envíos de dinero de los emigrantes, individual y colectivamente, redimían foros, pagaban deudas, edificaban y sostenían escuelas y otras obras públicas (desde lavaderos hasta traídas de aguas), promovían diarios y revistas, financiaban sindicatos agrícolas y cooperativas (NÚÑEZ SEIXAS, 2009).

En definitiva, dan cuenta de que hubo un tiempo en el que los emigrantes ayudaron a la regeneración de Galicia y a su modernización. De hecho, uno de los factores que posibilitaron el surgimiento de un tejido asociativo local de ámbito gallego en ciudades como Buenos Aires o La Habana, fue la interrelación entre movilización política y social a nivel local en Galicia durante el primer tercio del siglo XX, y su traslación a los emigrados, que jugaban un papel principal en esa dinámica de cambio social y acción colectiva. Ese afán de "mejoramiento y engrandecimiento moral, material y político" (como lo denominaba en 1916 la Sociedad Fomento de Porriño y su Distrito), ${ }^{22}$ aflora, por ejemplo, en la declaración con la que principia en febrero de 1910 el Libro de Actas del Comité Auxiliar de las Sociedades Agrícolas de Puenteareas en Buenos Aires:

\begin{abstract}
Considerando.
Que muchos de los males que aquejan a nuestra región tienen su origen en la indeferencia y apatía, causas de la ignorancia en la que nuestra vida tanto social como política se desenvuelve dentro de un individualismo egoísta perjudicial a los intereses colectivos, y la primer base para la creación del tipo del cacique de nuestros pueblos al cual los aldeanos creen un semidios, por estas consideraciones y otras muchas que sería obvio enumerar[,] entre ellas el fetichismo religioso que se cultiva en los campos a base de explotación [...]. Constatadas estas causas, y además siendo la única fuente de trabajo de nuestra región la agricultura[,] debiendo salir de los agricultores todo trabajo de regeneración para que sea eficaz y de positivas ventajas: por estas Consideraciones es que un núcleo de ciudadanos cree una obra útil a nuestra región el crear el mayor número posible de sociedades agrícolas en las aldeas[,] para desinfectar la atmósfera viciosa en que se desenvuelven las autoridades de los ayuntamientos rurales[,] primer peldaño de la política nacional[,] y concurrir por todos los medios a su alcance a la elevación intelectual de los labradores. por estas Consideraciones se funda en Buenos Aires El Comité de las Sociedades Agrícolas de Puenteareas. ${ }^{23}$
\end{abstract}

22 Citado en Núñez Seixas (2001, p. 378).

23 Archivo FAGA-MEGA, Fondo Centro Renovación de Puenteareas, Subfondo Comité Auxiliar de las Sociedades Agrícolas de Puenteareas en Buenos Aires, Libro de Actas, p. 2. 
Hemos dicho que hasta ahora los estudios migratorios referidos a la inmigración gallega en la Argentina se han centrado por lo general más en el período que antecede a la Primera Guerra Mundial que en el de entreguerras. De igual modo, el número de los dedicados a éste último aventajan a los que toman como marco de estudio lo sucedido a partir de 1946. Sin embargo, puesto que la FSG se constituyó en 1921, y que tanto ella como las múltiples microsociedades a las que coaligó vivieron su período álgido entre la tercera y la octava década del pasado siglo, la documentación conservada por la FAGA hace posible introducir ciertas correcciones a la antedicha tendencia.

En primer lugar, como ya anticipáramos, permite ahondar en la memoria de cómo los inmigrantes gallegos se movilizaron en apoyo de la II ${ }^{a}$ República española durante la Guerra Civil de 1936 a 1939. La Federación, reunificada a comienzos de 1937, dio vida a organismos específicos para apoyar la causa de la España leal (la Agrupación Gallega de Ayuda al Frente Popular, luego Central Gallega de Ayuda el Frente Popular Español, Central Gallega de Ayuda a los Refugiados Españoles y, finalmente, Sección Solidaridad de la FSG), de los cuales se conservan sus libros de actas y otros papeles, elaborados entre 1937 y 1949. Desde luego, el rico epistolario de la FSG constituye también una fuente ineludible para este tema (sobre todo para aquellos interesados en las personalidades destacadas), puesto que en él pueden hallarse cartas enviadas a y recibidas de múltiples representantes del republicanismo español, el galleguismo, etc., cuyos nombres resultaría ocioso reproducir aquí. ${ }^{24}$ Del mismo modo, existen otros fondos y sub-fondos correspondientes tanto a la Coordinación de Organismos Republicanos Españoles (COER), como a algunos de los comités que muchas de las sociedades federadas crearon con fines similares. Este es, por ejemplo, el caso del Comité de Castroverde de Ayuda al Frente Popular Español, del Comité Pro Ayuda a España de la Sociedad Hijos del Ayuntamiento de Carnota, o del Comité de Ayuda a la República Española del P. J . de Quiroga. ${ }^{25}$ Y documentos que nos muestran que hubo quienes, yendo aún más lejos, optaron por regresar a la Península y participar de lleno en el conflicto, haciendo gala de un grado de compromiso que, en ocasiones, les costó la vida. Tal fue la suerte de Amador Iglesias Gallego, que entre abril de 1936 y febrero de 1938 fuera miembro del Centro Renovación de Puenteareas,

${ }^{24}$ En ocasiones, también la correspondencia de las pequeñas sociedades federadas puede ofrecer algún documento de este tipo. Véase, por ejemplo, la carta de Dolores Ibarruri (a) "La Pasionaria", conservada en el Fondo Centro Renovación de Puenteareas, Correspondencia enviada y recibida, 1935-1939.

${ }^{25}$ Así, por ejemplo, entre 1940 y 1941 el Comité de Castroverde, Baleira y Fonsagrada de Ayuda al Frente Popular Español se interesó por la suerte de varias personas de estos municipios internadas en campos franceses. Archivo FAGA-MEGA, Fondo Comité de Castroverde, Baleira y Fonsagrada de Ayuda al Frente Popular Español, Refugiados. 
institución de la que se dio de baja antes de trasladarse a Barcelona. ${ }^{26}$ La mala fortuna quiso que, a poco dellegar a la ciudad catalana, lo encontrase la muerte:

\begin{abstract}
$\mathrm{Al}$ acusaros recibo, tengo que manifestaros, con profundo dolor, que el amigo AMADOR IGLESIAS, ha sido víctima de uno de los cobardes bombardeos que la aviación facciosa realizó los días 17 y 18 de los corrientes, sobre esta ciudad. Cuando apenas llevaba entre nosotros unos 8 o 10 días y dentro de pocos empezaría a prestar servicios en la Sub-secretaría de Armamentos como Practicante, la metralla traidora y cobarde de los enemigos de España, arrebató la vida de tan buen camarada cuyo entusiasmo y patriotismo había puesto al servicio de nuestra causa. ${ }^{27}$
\end{abstract}

Desde luego, los fondos pertenecientes al CRE constituyen otra vía de entrada, tanto al estudio del republicanismo español en la Argentina, como al de la Guerra Civil y al exilio. Esta institución, nacida en 1904, y que durante décadas mantuvo una importante actividad político-cultural a través de su Comisión de Cultura (luego denominada Ateneo Pi y Margall) y su periódico (España Republicana), editando libros a través de su propio sello editorial, sosteniendo una Biblioteca y Hemeroteca, así como también un programa radial durante las décadas de 1950 y 1960, se extinguió a finales de los años 80. Las 90 cajas y cinco cajones-ficheros que entonces recibiera la FAGA incluyen, entre otros valiosos documentos (y libros), ${ }^{28}$ no menos de 5.000 solicitudes de ingreso originales labradas entre 1914 y 1985, correspondientes tanto al propio CRE como a las agrupaciones Navarra Republicana y La Tierrina Leal (asturiana), y que, gracias a los datos filiatorios en ellos consignados, permiten reconstruir el perfil socioprofesional y político de los miembros de uno y otras. ${ }^{29}$

$\mathrm{Si}$ bien la mayor parte del resto de los fondos del archivo de la FAGA-MEGA refieren a cuestiones en apariencia menos impactantes, los mismos permiten abordar algunos de los temas fundamentales para la comprensión del proceso de integración de cualquier colectivo inmigrante. Piénsese, por ejemplo, en el largo debate que la historiografía argentina sobre las migraciones sostuvo en relación a si la Argentina del

${ }^{26}$ Archivo FAGA-MEGA, Fondo Centro Renovación de Puenteareas, Libro de Socios, p. 11.

${ }^{27}$ Carta de Ramón T. Fernández al Centro Renovación [de Puenteareas], Archivo FAGA-MEGA, Fondo Centro Renovación de Puenteareas, Correspondencia enviada y recibida.

${ }^{28} \mathrm{Si}$ bien no es este el lugar indicado para una descripción pormenorizada del contenido de aquellas cajas, no es posible pasar por alto la existencia, por ejemplo, de documentación perteneciente a la Cámara de Comercio Republicana Española, una entidad gremial creada por Manuel Puente (mecenas del político exiliado gallego Alfonso Daniel Rodríguez Castelao y de varios emprendimientos culturales de la colectividad galaica en la Argentina), que existió entre 1938 y 1945.

${ }^{29}$ Nombres y apellidos, fecha y lugar de nacimiento, dirección en la Argentina, filiación política actual y anterior, etc. 
pasado había sido una sociedad acrisolada o plural. ${ }^{30}$ En él, y gracias al uso de indicadores cuantificables y comparables, sus participantes ahondaron en tres indicadores que el modelo de Milton M. Gordon relaciona con el nivel de la asimilación estructural informal: pautas matrimoniales, residenciales y participación en asociaciones voluntarias (GORDON, 1964, p. 60-83) Vale la pena reflexionar sobre las posibilidades que el archivo de la FSG ofrece para el tratamiento de algunos de esos indicadores, y también de otros aspectos, como la composición del stock gallego en la Argentina y su inserción socioprofesional.

Antes, sin embargo, es conveniente introducir algunas prevenciones, relacionadas con el grado de representación de quienes se asocian a instituciones del tipo de las agrupadas en la FSG. ¿Cuán representativas del total de la población gallega en un área determinada pueden ser? Los acercamientos indirectos con que contamos indican que, en el mejor de los casos, las asociaciones gallegas que reproducían como marco de referencia ámbitos de relación e interacción social de origen de los inmigrantes inferiores a la provincia, podían llegar a nuclear entre un 33 y un $50 \%$ de sus connaturales emigrados en Buenos Aires, pero también que en otros casos los porcentajes podían ser muy inferiores. ${ }^{31}$ Por otra parte, como sostuviera hace ya tiempo Samuel Baily en sus estudios sobre los inmigrantes italianos en Nueva York y Buenos Aires, no debe perderse de vista que, al menos en dos aspectos, este tipo de instituciones no suelen estar integradas por inmigrantes "típicos": en ellas se encuentran sobrerrepresentados los segmentos más "estables" de la comunidad inmigrante (como lo indica el mismo hecho de que se asociaron a las entidades de corte étnico), y también los económicamente más prósperos (medido esto por su ocupación) (BAILY, 1996, p. 271-272). En consecuencia, deben evitarse los pasajes indiscriminados de lo observado en dicha colectividad a la totalidad de la colonia. ${ }^{32}$

Teniendo en cuenta estas prevenciones, veamos primero el tema de los patrones residenciales. Aunque ha pasado ya un cuarto de siglo desde que, en otro trabajo pionero, el mismo Baily (1985) diera paso a una sucesión de estudios sobre el tema (realizados a partir de una pluralidad de fuentes como los periódicos comunitarios, los

30 En la Argentina, el análisis de la naturaleza de su sociedad dio como resultado un fructífero debate que, entre la segunda mitad de la década de 1970 y mediados de la de 1990, se articuló en términos de una polaridad crisol de razas versus pluralismo cultural. Para un balance historiográfico sobre algunos de los períodos históricos, épocas o problemas de los estudios sobre migraciones en la Argentina, vid. Devoto y Otero (2003).

${ }^{31}$ Como la Sociedad Cultural El Pino, que en 1930 confesaba no reunir más que a un escaso 6,6 \% de sus convecinos en la ciudad. Vid. Núñez Seixas (2001, p. 373).

32 No obstante, en el caso de la inmigración gallega en la Argentina de la segunda posguerra, la dramática disminución de la tasa de retorno (en relación con lo sucedido antes de 1930) reduce sensiblemente el riesgo de subrepresentar a los emigrantes más "móviles" (aquellos que regresan a su tierra de origen). 
datos del registro civil y las cedulas censales), los registros de socios de las sociedades étnicas continúan siendo una valiosa herramienta para suplir, aunque sólo sea a través de estudios de caso, algunas de las lagunas de las fuentes oficiales argentinas éditas e inéditas. Como recordara Alicia Bernasconi, por lo general, aquellas que abarcan al conjunto de la población (como es el caso de las "cédulas censales") tienden a ser escasas en detalles particularizantes, mientras que, por el contrario, aquellas más amplias en cuanto al tipo de información que brindan (como la provincia o comuna de nacimiento) suelen ser más restringidas en el universo de emigrantes que abarcan (BERNASCONI, 1995, p. 192). ${ }^{33}$ El archivo de la FAGA-MEGA no sólo conserva más de 40 registros de socios correspondientes a un mínimo de 16 sociedades, sino también otras tantas series de solicitudes de ingreso, como las formadas por las 572 fichas de la Sociedad del Ayuntamiento de Castro Verde y sus Contornos (fundada como Sociedad Hijos de Montecuveiro y su Distrito), labradas entre 1927 y 1957, las 608 de Sociedad de Residentes del Municipio de Porriño confeccionadas entre 1938 y 1965, etc. En casi todos los casos, esas fichas consignan el nombre y apellido del nuevo socio (y el de quien lo presentó), su edad, nacionalidad, provincia, ayuntamiento y pueblo (sic) de nacimiento, su profesión y domicilio en la Argentina. ${ }^{34}$ La posibilidad de realizar comparaciones entre los diferentes estudios de caso, permite ilustrar los variados patrones residenciales que unos y otros grupos comarcales, municipales y parroquiales desarrollan. En algunas ocasiones, incluso, la existencia de más de un recuento en un mismo registro de socios permite observar también -someramente- la movilidad espacial del grupo. 35

A la vista de la información contenida en este tipo de documentos, resulta evidente que el de la inserción socioprofesional es otro de los indicadores para los que, en combinación con otras fuentes (tales como las actas de los registros civiles que contienen datos de ocupación, o los registros de personal de una o varias fábricas) las fichas de ingreso y/ o los registros de socios pueden proporcionar algún paliativo a las carencias de las fuentes argentinas. ${ }^{36}$ A grandes rasgos, el análisis de la composición

33 Por ejemplo, debido al alto nivel de agregación de sus datos resultan sumamente pobres los censos nacionales, provinciales y municipales de población argentinos, un hecho agravado por la destrucción y/ o pérdida de sus las cédulas posteriores a 1895.

${ }^{34}$ Asimismo, muchas de estas fichas requieren la firma del solicitante, por lo que permiten aproximarse (bien que de un momo no muy seguro) al tema de la alfabertización.

${ }^{35}$ Tal es el caso del de la Agrupación Cultural y Recreativa Residentes de Galicia en Lanús.

36 El tema de la inserción socioprofesional de los migrantes (y el de su movilidad social) adolece de graves dificultades en relación con las fuentes disponibles para su tratamiento, sea desde un enfoque macro basado en datos censales éditos, sea desde aquellos otros que realizan un seguimiento temporal de los individuos a través de las planillas originales de los censos. El dato "ocupación" presenta serios límites de cobertura y confiabilidad debido a la existencia de ocupaciones sumamente generales o sin un status definido, y al casi total silencio sobre las ocupaciones femeninas. Pero, además, para quien pretenda analizar este indicador exclusivamente 
social de los miembros de las asociaciones microterritoriales gallegas, revela que las mismas se componían por lo general de empleados y dependientes de comercio, seguidos por comerciantes y obreros manuales con y sin cualificación. ${ }^{37}$ Mismo las 323 solicitudes de ingreso de varones gallegos que entre 1944 y 1958 se inscribieron en Agrupación Cultural y Recreativa Residentes de Galicia en Lanús, muestran la existencia de un porcentaje de "empleados", comerciantes y "trabajadores artesanos" muy superior a la media de la colonia gallega en esa zona del primer cordón sur del Gran Buenos Aires y, al mismo tiempo, una presencia relativamente escasa de trabajadores urbanos con y sin cualificación, y la práctica inexistencia de marineros (que, sin embargo, resultan claramente perceptibles a partir de otras fuentes más amplias, como el Registro General de Matrícula del Consulado General de España en Buenos Aires labrado entre 1939 y 1960, que también contienen datos de la ocupación). ${ }^{38}$ Mas, en cualquier caso, esta conclusión general no debellevar a ignorar la existencia de matices importantes entre las diferentes instituciones.

Otro ítem que la documentación elaborada por las microsociedades nucleadas en la FAGA también nos permite abordar en ocasiones, es el de la composición del stock gallego en un punto determinado del país. Así, por ejemplo, las más de 800 solicitudes de ingreso labradas por Residentes de Galicia en Lanús entre 1944 y 1958 ofrecen, debido a la pretensión de la institución de nuclear a todos los gallegos residentes en aquel municipio bonaerense, una buena aproximación a la composición provincial del colectivo galaico en él. ${ }^{39}$ En otros casos, la utilidad de la fuente radica en la posibilidad de visualizar el diferente peso relativo que unas y otras parroquias o ayuntamientos pudieron tener en la conformación de las sociedades de ámbito municipal o comarcal.

El carácter nominativo de este tipo de fuentes (es decir, el hecho de que contienen información individual sobre una cantidad sustancial de gente) permite también la reconstrucción de familias, círculos de sociabilidad y, en definitiva, del vasto universo social de estas personas. Así, de su análisis suele desprenderse la existencia de redes microsociales de vecindad y parentela operantes en el lugar de destino, proximidad residencial y/ o de lugar de trabajo, y también una combinación de intereses en su

en relación con un grupo regional determinado, se añaden dos problemas más. En primer lugar, dado que estas fuentes no discriminan la región de origen, resulta imposible desligar con seguridad la información referida a quienes nacieron en Galicia de la que corresponde al resto de los españoles. Segundo, las últimas cédulas censales disponibles (1895) corresponden a un período anterior al momento álgido de la inmigración gallega en el país, situado entre 1904 y 1913.

37 En su análisis comparativo de los registros de socios de cuatro sociedades microterritoriales gallegas de Buenos Aires, Núñez Seixas (2001, p. 365-367) utiliza dos (el Centro Renovación de Puenteareas y la Unión Progresista del Distrito de Covelo) pertenecientes a la FSG.

38 Cfr. Archivo FAGA-MEGA, Fondo RGL, Solicitudes de ingresos de socios, 1-810; Farías (2010, p. 616-621).

39 Vid. Archivo FAGA-MEGA, Fondo RGL, Solicitudes de ingresos de socios, 1-810; 
liderazgo entre notables y activistas políticos. Sin embargo, quizás sea la utilidad crucial de este tipo de fuentes el hecho de que identifican tanto el lugar de nacimiento de esas personas como su domicilio en la Argentina y, una vez establecida la vinculación entre origen y destino, dejan abierta la posibilidad de expandir nuestra base documental incorporando, a manera de eslabones, datos adicionales obtenidos a partir de distintas fuentes labradas en uno y otro lugar. ${ }^{40}$

El estudio de las formas de sociabilidad de los colectivos emigrantes constituye, indudablemente, uno de los ítems más importantes dentro del campo de los estudios migratorios. Pero, como nos lo recuerdan Fernando Devoto y Hernán Otero, el indicador del asociacionismo étnico permite también abordar las estrategias de las elites en la construcción de los consensos y de la conciencia social en cada colectividad (DEVOTO; OTERO, 2003, p. 195). ${ }^{41}$ Porque, más allá de su aparente esterilidad, las actas de asamblea y de comisión directiva facilitan una aproximación a los discursos de las elites, y desde ella a una reflexión de la cuestión de la identidad, y de la relación de la dirigencia étnica con la masa de inmigrantes anónimos. ${ }^{42}$ Por otra parte, el análisis de las estrategias de la dirigencia comunitaria, junto al de los diferentes círculos concéntricos de identificación local, de las cuestiones político-ideológicas y de los mismos avatares de los flujos migratorios, permitirá entender las múltiples ampliaciones, escisiones y - sobre todo a partir de la década de 1930- fusiones entre las diferentes sociedades cuyos fondos integran el acervo documental de la FAGA.

Otro tema merecedor de la atención de los investigadores, es el del importante papel que tanto la Federación como muchas de sus sociedades adjudicaron a la formación cultural de sus miembros. Al respecto, no sólo existen los numerosos documentos generados por la sección cultural de la FAGA, el Ateneo Curros Enríquez (de los que se conservan sus libros de actas, correspondencia enviada y recibida, textos de audiciones radiales, etc.), sino también los libros y cuadernos pertenecientes a las bibliotecas de entidades microterritoriales tales como la Sociedad Unión Residentes de Entrimo y Lovios, Fomento de Porriño y su Distrito, Unión Hijos de El Grove, etc.

40 Un buen ejemplo de este tipo de metodología es la experiencia de Baily (1996) con los emigrantes de la localidad italiana de Agnone a la de Pueblo, en Colorado (Estados Unidos de Norteamérica). Véase particularmente la Tabla 1. En el caso de los españoles en Buenos Aires entre 1850-1930, es de referencia el modélico trabajo de Moya (2004).

${ }^{41} \mathrm{Al}$ respecto, véase, por ejemplo, el excelente estudio de Núñez Seixas (2002, p. 101-207) sobre el imaginario de los gallegos en la Argentina.

42 El liderazgo étnico constituye, indudablemente, uno de los puntos de referencia inexcusables para el estudio de un colectivo migratorio determinado. Véanse, por ejemplo, los diferentes trabajos reunidos en Bernasconi y Frid (2006). Para este tema, desde luego, resulta fundamental el análisis de la prensa étnica. Desde 1922, la FSG contó con un medio de prensa propio (El Despertar Gallego, que en 1930 mudó su nombre por el de Galicia), inclaudicable órgano de difusión de los gallegos que desde la emigración argentina apoyaban la causa republicana y de redención de Galicia, íntegramente conservado en el Hemeroteca de la FAGA-MEGA. 
Y, aunque sin duda resulta de lectura árida, la abundante documentación contable que se conserva permitirá, eventualmente, el estudio de la gestión financiera de estas entidades, y de la relación de está con sus variados fines.

¿Qué decir de la alimentación y el esparcimiento? No existen dudas respecto del destacado papel que las omnipresentes fiestas, almuerzos, pic-nics (fiestas campestres), etc., de las sociedades gallegas jugaron en la pervivencia de las solidaridades locales. ${ }^{43}$ En unas y otros se combinaban elementos tradicionales (gaiteros, alimentos del país, etc.) con otros propios de la sociedad de acogida (música criolla, asados), representando tanto una oportunidad para la recreación de los ambientes culinarios típicos y la música propia, como también para su hibridación. En este tipo de convivencias, en efecto, se refleja cierta armoniosa mixtura de las costumbres alimentarias de los inmigrantes con los nuevos hábitos adquiridos, de manera que tanto en la comida como en la música se produce una especie de sincretismo entre las costumbres de los inmigrantes y las presentes en la sociedad receptora. Las omnipresentes comisiones de fiestas (para las que por regla general se designaba a los socios femeninos), y los documentos que las mismas generaron o acumularon a lo largo de décadas, constituyen otras tantas ventanas a este tipo de dinámicas clave para la sociabilidad del grupo. Pues aunque la organización de los festivales solía saldarse con pérdidas para la caja social, ninguna entidad renunciaba a celebrar anualmente un buen número de esas reuniones, debido a su alta "rentabilidad" en términos comunitarios y asociativos: en ellas se producía la recreación afectiva y simbólica del espacio social de origen. Por otra parte, tales acontecimientos, junto a las también numerosas comisiones "de damas y señoritas", representan prácticamente la única ventana a la por lo general "invisible" participación femenina en las instituciones de la diáspora gallega. Pues si bien el papel de la mujer en la mayoría de ellas la colocó en una situación de subordinación al hombre (rara vez ocupaban puestos en las comisiones directivas), resultaba en cambio sumamente importante en su función de organizadora de actos sociales y veladas festivas.

El archivo de la FAGA-MEGA constituye también una buena vía de entrada al problema de la identidad lingüística, sobre todo para el período posterior a 1936, hasta hoy poco explorado. ${ }^{44} \mathrm{Al}$ legar a Buenos Aires, los inmigrantes gallegos se enfrentaron a la necesidad de adaptarse a las normas y pautas de una sociedad distinta y cuyo

\footnotetext{
${ }^{43}$ Sobre la fiesta y el teatro gallego en Buenos Aires entre finales del siglo XIX y el primer tercio del XX, vid. Núñez Seixas (2002, p. 245-316). Por el momento, continuamos huérfanos de estudios que repliquen el trabajo realizado por este autor para la segunda y tercera década del siglo XX. Sobre la comida, algunos breves apuntes en Cagiao Vila (1999, p. 131-135); Farías (2009, p. 86-91).

${ }^{44}$ Para el período anterior a 1940. Núñez Seixas (2001, p. 209-243). Una aproximación a la situación imperante a partir de la última oleada migratoria en Gugenberger (2001).
} 
idioma oficial es el castellano, la misma lengua dominante en Galicia, de donde muchos llegaban monolingües en gallego, pero cargando el estigma de hablar un habla que era sinónimo de tonto e ignorante. A ello se añade la presión social del entorno castellanohablante o, cuando menos, la necesidad de comunicarse en un idioma franco con los inmigrantes de otras nacionalidades que tampoco conocían la lengua oficial del país. De este modo, en su proceso de incorporación a la sociedad porteña acabaron por entrelazarse la motivación instrumental y la integradora. El fuerte deseo de superación, de ascender socialmente y de brindar a sus hijos un futuro mejor, son factores que también fomentan la falta de transmisión intergeneracional de idioma gallego. Por ello, si puede suponerse que el mismo siguió siendo empleado de acuerdo con el código de "lengua de solidaridad" que imperaba en el medio rural gallego, y continuó hablándose en el seno de las redes sociales informales y familiares, en las fiestas y, naturalmente, en los momentos de esparcimiento en los centros y sociedades étnicas, su uso público "formal" debió ser muy reducido. En el primer tercio del siglo $\mathrm{XX}$, el habla galaica se halla prácticamente ausente de la documentación interna y de las memorias editadas por las asociaciones de emigrantes y, con anterioridad a la década de 1920, rara vez era empleada en público en los actos de la colectividad. De hecho, su uso en circunstancias "formales" causaba extrañeza, y la reacción de la elite no solía ser positiva cuando el empleo del mismo superaba las funciones secundarias, "permitidas" y más o menos cómico-festivas. La diglosia funcional asociaba y limitaba el uso escrito del gallego a la gastronomía y diversión, junto con la evocación de la comarca de origen. La actitud que la FAGA y las sociedades federadas tuvieron al respecto en los años posteriores a 1936 constituye, indudablemente, otro tema abierto a la investigación.

\section{A manera de Cierre}

$\mathrm{Si}$ en las últimas décadas, los estudios migratorios han contribuido de manera decisiva a modificar las interpretaciones de la historia de los países sobre las que han reflexionado, ello se debe a que la inmigración en sí constituye un tema de investigación vasto y apasionante, sino porque supone, además, una vía de entrada a problemas más generales de la sociedad.(DEVOTO; OTERO, 2003). Constreñidos por lo reducido del espacio con el que contamos no hemos profundizado en ello. Confiamos, sin embargo, en que nuestra somera descripción y análisis de la composición y características del archivo de la FAGA-MEGA, sirva al menos para dejar en claro el valor que sus fondos documentales poseen para el tratamiento de diversos aspectos de la inmigración y el exilio gallego en la Argentina. Aspectos que, desde luego, exceden 
largamente su obvia utilidad para la reconstrucción de la vida societaria de la comunidad emigrada o, en el caso de entidades altamente politizadas como la FAGA, de la trayectoria de aquellos personajes relevantes (políticos, intelectuales, artistas) de la emigración y el exilio.

\section{Referencias}

BAILY, Samuel. Patrones de residencia de los italianos en Buenos Aires y Nueva York: 1880-1914. Estudios Migratorios Latinoamericanos, Buenos Aires, n. 1, p. 8-47, Dic. 1985.

BAILY, Samuel. Posibilidades y problemas del cruzamiento de registros nominativos en el estudio del proceso migratorio italiano. Estudios Migratorios Latinoamericanos, Buenos Aires, n. 33, p. 269-285, Ago. 1996.

BERNASCONI, Alicia. Aproximación al estudio de las redes sociales migratorias a través de las listas de desembarco: posibilidades y problemas. BJ ERG, María; OTERO, Hernán. Inmigración y redes sociales en la Argentina moderna. Tandil: CEMLAIEHS, 1995. p. 191-202.

BERNASCONI, Alicia; FRID, Carina Frid. De Europa a las Américas: dirigentes y liderazgos (1880-1960). Buenos Aires: Biblos, 2006.

CAGIAO VILA, Pilar. A vida cotiá dos emigrantes galegos en América. In:

Galegos en América e americanos en Galicia. Santiago de Compostela: Xuntā de Galicia, 1999. p. 115-135.

CAGIAO VILA, Pilar. Muller e emigración. Santiago de Compostela: Xunta de Galicia, 1997.

CAGIAO VILA, Pilar; NÚÑEZ SEIXAS, Xosé Manoel. Os galegos e o Río da Prata. A Coruña: Arrecife Edicións, 2007.

CHILOTEGUY, Miguel. Plan de recuperación documental. In: ENCUENTRO DE BIBLIOTECAS DE COLECTIVIDADES, 5., 2010, Buenos Aires. Anales... Buenos Aires, 2010.

CLEMENTI, Hebe. Inmigración española en la Argentina. Buenos Aires: Oficina Cultural de la Embajada de España, 1991.

CUPEIRO, Bieito. A Galiza de alén mar. Sada: Ediciós do Castro, 1989.

DA ORDEN, María Liliana. Inmigración española, familia y movilidad social en la Argentina moderna: una mirada desde Mar del Plata (1890-1930). Buenos Aires: Biblos, 2005.

DE CRISTÓFORIS, Nadia; FERNÁNDEZ, Alejandro. Las migraciones españolas a la Argentina: Variaciones regionales (siglos XIX y XX). Buenos Aires: Biblos, 2008. 
DEVOTO, Fernando. El revés de la trama: políticas migratorias y prácticas administrativas en la Argentina (1919-1949). Desarrollo Económico, Buenos Aires, v. 41, n. 162, p. 281-303, Jul./Sep. 2001.

DEVOTO, Fernando; FERNÁNDEZ, Alejandro. Mutualismo étnico, liderazgo y participación política: algunas hipótesis de trabajo. In: ARMUS, Diego. Mundo urbano y cultura popular. Buenos Aires: Sudamericana, 1990. p. 129-152.

DEVOTO, Fernando; OTERO, Hernán. Veinte años después: una lectura sobre el pluralismo cultural y la historia nacional en la historiografía argentina. Estudios Migratorios Latinoamericanos, Buenos Aires, n. 50, p. 181-227, 2003.

DÍAZ, Hernán M. Historia de la Federación de Sociedades Gallegas: identidades políticas y prácticas militantes. Buenos Aires: Biblos, 2007.

DUARTE, Ángel. La República del emigrante: la cultura política de los españoles en la Argentina, 1875-1910. Lleida: Milenio, 1998.

FARÍAS, Ruy. Alimentación, sociabilidad y lenguaje: a propósito de la identidad gallega en Buenos Aires en las primeras seis décadas del siglo XX. Gavieiro da nosa identidade, Buenos Aires, ano 3, n. 3, p. 70-97, 2009.

FARÍAS, Ruy. Buenos Aires Gallega: inmigración, pasado y presente. Buenos Aires: CPPHC, 2007.

FARÍAS, Ruy. La inmigración gallega en el sur del Gran Buenos Aires, 1869-1960. Santiago de Compostela: Universidade de Santiago de Compostela, 2010. CD-ROM.

FERNÁNDEZ VARGAS, Valentina. Análisis cuantitativo. In: VIVES AZANCOT, Pedro A.; VEGA, Pepa; OYAMBURU, J esús (coord.). Historia general de la emigración española a Iberoamérica. Madrid: Historia 16, 1992. v. 1. p. 579-614.

FERNÁNDEZ VICENTE, María J osé. En busca de la legitimidad perdida: la política de emigración del régimen franquista, 1946-1965. Estudios Migratorios Latinoamericanos, Buenos Aires, n. 56, p. 3-29, Abr. 2005.

FERNÁNDEZ, Alejandro; MOYA, J osé Carlos. La inmigración española en la Argentina. Buenos Aires: Biblos, 1999.

GALANTE, Miguel Alberto. Conflictos y alternativas en las políticas migratorias del primer peronismo: del nacionalismo racista a la planificación económico-social (19431949). Buenos Aires: Universidad de Buenos Aires, 2005.

GORDON, Milton M. Assimilation in American Lyfe: the role of race, religion and national origins. New York: Oxford University Press, 1964.

GUGENBERGER, Eva. Identidad, conflicto lingüístico y asimilación: observaciones acerca de la lengua gallega en Buenos Aires. In: NÚÑEZ SEIXAS, Xosé Manoel. La Galicia Austral: la inmigración gallega en la Argentina. Buenos Aires: Biblos, 2001. p. 251-277.

LOJ O, María Rosa. Los "gallegos" en el imaginario argentino: literatura, sainete, prensa. A Coruña: Fundación Pedro Barrié de la Maza, 2008. 
MARQUIEGUI, Dedier. La inmigración española de masas en Buenos Aires. Buenos Aires: CEAL, 1993.

MOYA, J osé C. Primos y extranjeros: la inmigración española en Buenos Aires, 18501930. Buenos Aires: Emecé, 2004.

NÚÑEZ SEIXAS, Xosé Manoel. ¿Para qué sirve conservar el patrimonio de la emigración?. 2009. A Grileira, n. 7. Disponible em: <http:// www.agrileira.com.ar/ agrilev/ 7C/ nota1c.htm>. Acesso em: 10 set. 2010.

NÚÑEZ SEIXAS, Xosé Manoel. A parroquia de alén mar: algunhas notas sobre o asociacionismo local galego en Bos Aires (1904-1936). Semata. Ciencias Sociais e Humanidades, Santiago de Compostela, n. 11, p. 345-379, 1999a.

NÚÑEZ SEIXAS, Xosé Manoel. Asociacionismo local y movilización sociopolítica: notas sobre los gallegos en Buenos Aires (1890-1936). In: MOYA, J osi C. La inmigración española en la Argentina. Buenos Aires: Biblos, 1999b. p. 195-233.

NÚÑEZ SEIXAS, Xosé Manoel. Emigrantes, caciques e indianos: o influxo sociopolítico da emigración transoceánica en Galicia (1900-1930). Vigo: Xerais, 1998.

NƯÑEZ SEIXAS, Xosé Manoel. La Galicia Austral: la inmigración gallega en la Argentina. Buenos Aires: Biblos, 2001.

NÚÑEZ SEIXAS, Xosé Manoel. O galeguismo en América, 1879-1936. Sada: Edición do Castro, 1992.

NÚÑEZ SEIXAS, Xosé Manoel. O inmigrante imaginario: estereotipos, identidades e representacións dos galegos na Arxentina (1880-1940). Santiago de Compostela: Universidade de Santiago de Compostela, 2002.

NÚÑEZ SEIXAS, Xosé Manoel; SOUTELO VÁZQUEZ, Raúl. As cartas do destino: Unha familia galega entre dous mundos, 1919-1971. Vigo: Galaxia, 2005.

PALAZÓN FERRANDO, Salvador. Capital humano español y desarrollo latinoamericano: evolución, causas y características del flujo migratorio (1882-1990). Valencia: Institut de Cultura "J uan Gil-Albert", 2005.

PALMÁS, Ricardo. A emigración galega á Arxentina. Sada: Ediciós do Castro, 1978.

PEÑA SAAVEDRA, Vicente. Éxodo, organización comunitaria e intervención escolar: la impronta socio-educativa de la emigración transoceánica en Galicia. Santiago de Compostela: Xunta de Galicia, 1991. 2. v.

PÉREZ-PRADO, Antonio. Los gallegos y Buenos Aires. Buenos Aires: La Bastilla, 1973.

QUIJ ADA MAURIÑO, Mónica. Política inmigratoria del primer Peronismo: las negociaciones con España. Revista Europea de Estudios Latinoamericanos y del Caribe, n. 47, p. 43-64, dic. 1989.

SANCHEZ ALONSO, Blanca. La inmigración española en Argentina: siglos XIX y XX. Columbres: Archivo de Indianos, 1992. 
SANCHEZ ALONSO, Blanca. Las causas de la emigración española, 1880-1930. Madrid, Alianza, 1995.

SÁNCHEZ-ALBORNOZ, Nicolás. Españoles hacia América: la emigración en masa, 1880-1930. Madrid: Alianza, 1988.

SCHWARZSTEIN, Dora. Entre Franco y Perón: memoria e identidad del exilio republicano español en Argentina. Barcelona: Crítica, 2001.

SENKMAN, Leonardo. Argentina, la segunda guerra mundial y los refugiados indeseables. Buenos Aires: GEL, 1991.

VÁZQUEZ GONZÁLEZ, Alejandro. La emigración gallega a América, 1850-1930. Santiago de Compostela: Universidade de Santiago de Compostela, 2000. CD-ROM.

VILANOVA RODRÍGUEZ, Alberto. Los gallegos en la Argentina. Buenos Aires: Ediciones Galicia, 1966.

VILLARES, Ramón; FERNÁNDEZ, Marcelino. Historia da emigración galega a América. Santiago de Compostela: Xunta de Galicia, 1996.

VIVES AZANCOT, Pedro A.; VEGA, Pepa; OYAMBURU, J esús (coord.). Historia general dela emigración española a Iberoamérica. Madrid: Historia 16, 1992. v. 2.

Colaboração recebida em 30/ 07/ 2010 e aprovada em 09/ 08/ 2010. 